\title{
Expression and Significance of miR-126 and miR-145 in Infertility due to Endometriosis
}

\author{
Fang Cheng1, Luyan Lu1, Huixia Wang1, Hong Cheng2 and Dawei Zhang1
}

\begin{abstract}
The aim of this study was to determine the expression and significance of miR-126 and miR-145 in endometrial tissues in the mid-luteal phase of patients with infertility due to endometriosis. It was an experimental study carried out from January 2016 to July 2017. A total of 47 infertile patients with endometriosis were selected as an endometriosis group, and classified as 22 cases $(46.81 \%)$ in stage I-II and 25 cases $(53.19 \%)$ in stage III-IV; while 47 women who were infertile due to male factors were selected as a control group. The research showed that the expression levels of miR-126 and miR-145 in endometriosis group were higher than those of the control group (both $p<0.001$ ). There was no significant difference in the expression levels of miR-126 and miR-145 between the I-II and III-IV patients ( $p=0.535$ and 0.809 , respectively). The expression levels of miR-126 and miR-145 in endometrial tissues of non-pregnant patients were significantly higher than those of pregnant patients (both $p<0.001$ ). In conclusion, the up-regulated expression of miR-126 and miR-145 in the endometrial tissues in the mid-luteal phase of patients may play a role in infertility due to endometriosis.
\end{abstract}

Key Words: Endometriosis, Infertility, Luteal phase, Endometrium.

Endometriosis is a hormone-dependent chronic disease in which endometrial tissue grows and infiltrates in areas other than the endometrium of the uterine cavity. The etiology of endometriosis is still unclear, but most scholars believe that the basic pathological processes in the formation of endometriosis include adhesion, invasion, and angiogenesis of endometrial stromal cells. ${ }^{1}$ Non-coding single stranded small molecule RNA (miRNA) is a microRNA that has been found in recent years to regulate gene expression and is about 17 to 24 nucleotides in length. Related data shows that a variety of miRNAs affect the apoptosis, invasion and angiogenesis of endometriosis, and may be involved in the pathophysiological process of endometriosis. ${ }^{2}$ Some scholars have screened for endometriosis related miRNAs, and found that there are differential expression profiles of miRNAs in ectopic endometrial tissues, especially the abnormal expression of miR-126 and miR-145. ${ }^{3}$ These evidences suggest that miR-126 and miR-145 may be involved in the pathogenesis and progression of endometriosis.

The objective of this study was to determine the expression and significance of miR-126 and miR-145 in

1 Department of Gynaecology and Obstetrics, The Third Affiliated Hospital of Henan University of Traditional Chinese Medicine, Zhengzhou City, 450004, China

2 Department of Rehabilitation, Henan Province Hospital of TCM, Zhengzhou City, 450002, China

Correspondence: Dawei Zhang, Department of Gynaecology and Obstetrics, The Third Affiliated Hospital of Henan University of Traditional Chinese Medicine, Zhengzhou City, 450004, China E-mail:fbteo8@163.com

Received: August 08, 2018; Accepted: January 16, 2019 endometrial tissues in the mid-luteal phase of patients with infertility due to endometriosis.

This experimental study was conducted at the Department of Gynaecology and Obstetrics, The Third Affiliated Hospital of Henan University of Traditional Chinese Medicine, Zhengzhou City, China, from January 2016 to July 2017. This study was approved by the hospital Ethics Committee. A total of 47 patients with endometriosis underwent laparoscopic surgery and confirmed by postoperative pathological examination, and needed treatment by in vitro fertilisation and embryo transfer after laparoscopic surgery were selected as an endometriosis group, excluding other causes of infertility such as hydrosalpinx and polycystic ovary syndrome. Forty-seven women who passed all tests and were infertile due to male factors such as obstructive azoospermia and severe oligospermia were selected as a control group. Both basic endocrine and chromosomal examinations of patients in the endometriosis group and the control group were normal, and there were no genital malformations, ovarian tumors, endocrine, immune or metabolic diseases, tuberculosis, liver and kidney diseases or history of rest of the family. In five weeks after embryo transfer, patients with the intrauterine gestation sac and cardiac pulsation detected by B-ultrasound were treated as clinical pregnancy. Two weeks after the embryo transfer, the endometriosis group was followed up to confirm the pregnancy.

In the mid-luteal phase, a specially designed endometrial device with a diameter of about $1 \mathrm{~mm}$ was used by clinically experienced doctors to enter the uterine cavity, reach the bottom of the uterus or the anterior or posterior wall of the uterus, and the endometrial tissue with a diameter of 1-2 mm was extracted. The total RNA 
was extracted from the endometrial tissues in the luteal phase of patients in the endometriosis group and control group with the TRIzol method. The extracted total RNA was quantified by UV spectrophotometer and reverse transcribed on a PCR machine using a miRNA reverse transcription kit. The reaction system was: total RNA 1 $\mu \mathrm{g}$, reverse transcriptase $2 \mu \mathrm{L}$, buffer $10 \mu \mathrm{L}, 0.1 \%$ bovine serum albumin (BSA) $2 \mu \mathrm{L}$ and $\mathrm{dH}_{2} \mathrm{O}$ (total 20 $\mu \mathrm{L}), 37^{\circ} \mathrm{C}$ for $60 \mathrm{~min}, 85^{\circ} \mathrm{C}$ for $5 \mathrm{~s}$. Real-time quantitative PCR was used to detect the expression levels of miR126 and miR-145 in endometrial tissues. Primers were designed by Primer 5.0 software. The forward primer of miR-126 was 5'-GGCTCGTACCGTGAGTAAT-3', and the reverse primer was 5'-GTGCAGGGTCCGAGGT-3'. The forward primer of miR-145 was 5'-ATGGTTCGTGGGG TCCAGTTTT CCCAG-3', and the reverse primer was 5'GTGTCGT GGAGTCGGCAATTC-3'. The amplification was performed on a PCR instrument using a miRNA quantitative PCR detection kit. The reaction system was: template cDNA $1 \mu \mathrm{L}$, primer $1 \mu \mathrm{L}$, Mix $12.5 \mu \mathrm{L}, \mathrm{dH} 2 \mathrm{O}$ $10.5 \mu \mathrm{L}$, pre-degeneration $95^{\circ} \mathrm{C}$ for $10 \mathrm{~s}, 95^{\circ} \mathrm{C}$ for $5 \mathrm{~s}$, $60^{\circ} \mathrm{C}$ for $20 \mathrm{~s}$, for 40 cycles, and 3 parallel experiments were conducted. Taking the expression of U6 as the internal control, the relative quantification method was used to calculate the $\triangle \mathrm{Ct}$ of the endometriosis group and the control group according to the formula $\triangle \mathrm{Ct}=\mathrm{Ct}$ target gene-Ct reference gene. The relative expression level of the target gene was $2-\triangle \Delta \mathrm{ct}$. The relative expression levels of 3 parallel experiments were recorded and the mean value was calculated.

Collected data were analysed using descriptive statistics by Statistical Package for Social Sciences (SPSS) version 25. Measurement data shows in Mean $\pm S D$. Independent sample t-test was used to compare the expression levels of miR-126 and miR-145 in the luteal phase of endometrial tissues of pregnant and nonpregnant women after treatment between groups and those in different stages of the disease. Enumeration data is represented by $n(\%)$. The $p<0.05$ means there is statistical significance in the difference.

Patients in the endometriosis group were 23-34 years old with an average age of $30.56 \pm 1.73$ years. Patients in the control group were 22-34 years old with an average age of $30.08 \pm 1.54$ years. According to the revised classification of endometriosis by the American Fertility Society in 1985, 47 patients in the endometriosis group were classified as 22 cases (46.81\%) in stage I-II and 25 cases $(53.19 \%)$ in stage III-IV.

The expression levels of miR-126 and miR-145 in endometrial tissues of the endometriosis group were higher than those of the control group (both $p<0.001$, Table I).

There was no significant difference in the expression levels of miR-126 and miR-145 between the I-II and III-IV patients ( $p=0.535$ and 0.809 , respectively, Table I).

After the assisted reproduction treatment, 16 cases (34.04\%) were pregnant and 31 cases $(65.96 \%)$ were not pregnant in the endometriosis group. The expression levels of miR-126 and miR-145 in endometrial tissues of non-pregnant patients were significantly higher than those of pregnant patients (both $p<0.001$, Table I).

Women with endometriosis suffer a delayed diagnosis by 6-12 years according to the usual experience. This delay increases the severity of the disease. Laparoscopy is the gold standard for the diagnosis of endometriosis. However, its accuracy rate is only $70 \%-75 \%$. The miRNAs can serve as diagnostic markers for disease in human circulation and body fluids such as serum, saliva, semen and urine, ushering in a new era of disease research. Similarly, miRNAs can be used as a non-invasive biomarker for early diagnosis and progression of endometriosis. Jia et al. found that the expression levels of 27 kinds of miRNAs in the serum of patients with endometriosis were significantly different from those of normal women with the gene chip method. 4 Related studies have suggested that adhesion-invasionangiogenesis describes the formation of endometriosis foci. The interaction of vascular endothelial growth factor and other angiogenic factors promotes the occurrence and development of endometriosis. miR-126 is a microRNA closely related to vascular endothelial cells and vascular functions. Studies have confirmed that miR-126 is involved in the angiogenesis and vascular remodeling during regulation and development, and guides the process of vascularization together with physiological stimulation and growth factor signaling. 5 The results of this study showed that the expression

Table I: Comparison of the expression levels of miR-126 and miR-145 in endometrial tissues.

\begin{tabular}{|c|c|c|c|c|c|}
\hline & \multirow[t]{2}{*}{$\mathrm{n}$} & \multicolumn{2}{|c|}{ miR-126 } & \multicolumn{2}{|c|}{ miR-145 } \\
\hline & & Mean \pm SD & $p$-value & Mean \pm SD & p-value \\
\hline \multicolumn{6}{|l|}{ Groups } \\
\hline Control group & 47 & $16.37 \pm 2.28$ & $<0.001$ & $4.16 \pm 1.75$ & $<0.001$ \\
\hline Endometriosis group & 47 & $58.51 \pm 3.46$ & & $6.52 \pm 2.63$ & \\
\hline \multicolumn{6}{|l|}{ Stages } \\
\hline$|-| \mid$ & 22 & $58.94 \pm 4.43$ & 0.535 & $6.46 \pm 1.97$ & 0.809 \\
\hline III-IV & 25 & $58.16 \pm 4.12$ & & $6.63 \pm 2.71$ & \\
\hline \multicolumn{6}{|l|}{ Category } \\
\hline Pregnant patients & 16 & $10.87 \pm 5.43$ & $<0.001$ & $1.12 \pm 0.15$ & $<0.001$ \\
\hline Non-pregnant patients & 31 & $63.44 \pm 8.03$ & & $7.37 \pm 3.28$ & \\
\hline
\end{tabular}


level of miR-126 in endometrial tissue of patients with endometriosis was higher than that of the control group; there was no significant difference in the expression level of miR-126 between patients in stage I-II and stage III-IV; and the expression level of miR-126 in the endometrial tissues of non-pregnant patients was significantly up-regulated compared with that of pregnant ones, suggesting the possible association of miR-126 with ectopic lesion angiogenesis.

miR-145 is one of the microRNAs found to be involved in gynecological diseases in recent years, and is associated with mutations in endometrial cancer cells. In addition, studies have shown that miR-145 can inhibit the proliferation, invasion and stem cell differentiation phenotype of ectopic endometrial cells in vitro by downregulating JAM-A, PAI-1 and some cell scaffold proteins. The results of this study showed that the expression level of miR-145 in endometrial tissue of patients with endometriosis was higher than that of the control group; there was no significant difference in the expression level of miR-145 between patients in stage I-II and stage $\mathrm{III}-\mathrm{IV}$; and the expression level of miR-145 in the endometrial tissues of non-pregnant patients was significantly up-regulated compared with that of pregnant ones, indicating that the change of miR-145 may be related to infertility in endometriosis. Adammek et al. have found that miR-145 inhibits the invasion of ectopic endometrial cells in patients with endometriosis by down-regulating plasma PAl-1.6 The specific mechanism and signaling pathway of miR-145 in the development of infertility due to endometriosis remain to be further studied.

The limitations of this study are that the sample size was small and due to lack of comprehensiveness, the correlation between miR-126 and miR-145 expression was not analysed, etc. Therefore, the relevant conclusions need to be further explored and improved.

In summary, the up-regulated expression of miR-126 and miR-145 in the endometrial tissues in the mid-luteal phase of patients may play a role in infertility due to endometriosis, which can be used as molecular markers for early diagnosis and prognosis of infertility due to endometriosis.

Acknowledgement: This study was supported by Special Subject Program Foundation of TCM Science Research of Henan Province (2017ZY1022, 2018ZY2010).

\section{REFERENCES}

1. Burney RO, Giudice LC. Pathogenesis and pathophysiology of endometriosis. Fertil Steril 2012; 98:511-9.

2. Abe W, Nasu K, Nakada C, Kawano Y, Moriyama M, Narahara H. Mir-196b targets c-myc and bcl-2 expression, inhibits proliferation and induces apoptosis in endometriotic stromal cells. Hum Reprod 2013; 28:750-61.

3. Bingbing Zheng, Xiangyang Xue, Yiping Zhao, Jing Chen, Chaoyi Xu, Ping Duan. The differential expression of microRNA143,145 in endometriosis. Iran J Reprod Med 2014; 12:555-60.

4. Jia SZ, Yang Y, Lang J, Sun P, Leng J. Plasma miR-17-5p, miR-20a and miR-22 are down-regulated in women with endometriosis. Hum Reprod 2013; 28:322-30.

5. Fish JE, Santoro MM, Morton SU, Yu S, Yeh RF, Wythe JD, et al. Mir-126 regulates angiogenic signaling and vascular integrity. Dev Cell 2008; 15:272-84.

6. Adammek M, Greve B, Kässens N, Schneider C, Brüggemann K, Schüring AN, et al. MicroRNA miR-145 inhibits proliferation, invasiveness, and stem cell phenotype of an in vitro endometriosis model by targeting multiple cytoskeletal elements and pluripotency factors. Fertil Steril 2013; 99:1346-55. 